\title{
Oncocytic adrenal tumour presenting as Cushing syndrome: rare presentation of a rare tumour Abhishek Singhai ${ }^{a}$, Subodh Banzal ${ }^{\mathrm{b}}$
}

aDepartments of Medicine, bendrocrinology, Sri Aurobindo Medical College, Indore, Madhya Pradesh, India

Correspondence to Abhishek Singhai, Department of Medicine, Sri Aurobindo Medical College, Indore, 453555, Madhya Pradesh, India

Tel: +91-0731-4231000; fax: +91-0731-4231010; e-mail: drabhisheksinghai@gmail.com

Received 29 July 2014

Accepted 23 August 2014

The Egyptian Society of Internal Medicine 2014, 26:184-185

\begin{abstract}
Oncocytic neoplasms are well recognized in organs such as the kidney, thyroid and salivary glands. They are seen rarely in other sites such as the adrenal cortex. To date, around 20 cases of oncocytic adrenocortical neoplasm have been reported; most of them were benign and nonfunctioning. We report the case of hypersecreting adrenocortical oncocytoma in a 52-year-old woman. The patient was tested because of cushingoid features. The patient underwent a laparotomy for right adrenal gland mass. The pathology report confirmed adrenocortical oncocytoma.
\end{abstract}

\section{Keywords:}

adrenal gland, cushing syndrome, oncocytoma

Egypt J Intern Med 26:184-185

(c) 2014 The Egyptian Society of Internal Medicine 1110-7782

\section{Introduction}

Hamperi [1] introduced the term 'oncocyte' in 1931 referring to a cell with abundant, granular, eosinophilic cytoplasm. Electron microscopic studies revealed that this granularity was due to mitochondria accumulation in the oncocyte cytoplasm [2]. Neoplasms composed predominantly or exclusively of this kind of cells are called 'oncocytic'. Such tumours have been described in the overwhelming majority of organs: kidney, thyroid and pituitary gland, salivary, adrenal, parathyroid and lacrimal glands, paraganglia, respiratory tract, paranasal sinuses and pleura, liver, pancreatobiliary system, stomach, colon and rectum, central nervous system, female and male genital tracts, skin and soft tissues [3]. Oncocytic adrenocortical neoplasms are rare, usually nonfunctioning tumours found predominantly in adults. The majority of those reported have followed a benign clinical course [4]. To date, around 20 oncocytic adrenocortical neoplasms have been described. We present the clinical, histologic and immunohistochemical features of oncocytic adrenocortical carcinoma that presented as Cushing syndrome.

\section{Case history}

A 52-year-old woman with history of hypertension and diabetes since 12 years was admitted with complaints of polyuria, generalized weakness, proximal muscle weakness, recurrent boils, weight gain, hirsutism and constipation. On examination, patient was febrile, pulse $82 / \mathrm{min}$, blood pressure 150/100 $\mathrm{mmHg}$ and had puffiness of face. Rest systemic examination was normal. Investigation showed haemoglobin11 gm/ dl, white blood cell count $10400 / \mathrm{mm}^{3}$, fasting blood glucose $217 \mathrm{mg} / \mathrm{dl}$, postprandial blood sugar $310 \mathrm{mg} / \mathrm{dl}$, $\mathrm{HbA}_{1 \mathrm{c}} 6.1$, serum creatinine $1.2 \mathrm{mg} / \mathrm{dl}$, serum sodium $137 \mathrm{mEq} / 1$, potassium $3.69 \mathrm{mEq} / 1$, total cholesterol 145 $\mathrm{mg} / \mathrm{dl}$, triglyceride $81 \mathrm{mg} / \mathrm{dl}$, liver function test normal, increased cardiothoracic ratio on chest radiography, left ventricular hypertrophy on ECG, $\mathrm{T}_{3} 0.54 \mathrm{ng} / \mathrm{ml}$, $\mathrm{T}_{4} 7.01 \mu \mathrm{g} / \mathrm{d}$, thyroid stimulating hormone $1.23 \mu \mathrm{IU} /$ $\mathrm{ml}$, serum cortisol (8 a.m.) $41.79 \mu \mathrm{g} / \mathrm{dl}$ (8.7-22.4), serum cortisol (after overnight $1 \mathrm{mg}$ dexamethasone suppression) $24.75 \mu \mathrm{g} / \mathrm{dl}(<10.00 \mu \mathrm{g} / \mathrm{dl})$, serum cortisol (after overnight $8 \mathrm{mg}$ dexamethasone suppression) $26.90 \mu \mathrm{g} / \mathrm{dl}$, serum dehydroepiandrosterone sulphate $4.50(56.20-282.90 \mu \mathrm{g} / \mathrm{dl})$, plasma adrenocorticotropic hormone less than $5.00 \mathrm{pg} / \mathrm{ml}$ and total testosterone $0.01 \mathrm{ng} / \mathrm{dl}$. On the basis of these investigations, patient was diagnosed as a case of Cushing syndrome. Ultrasonographic abdomen showed hypoechoic lesion over the right adrenal gland. MRI abdomen showed heterogeneously hyperintense soft tissue lesion over the right adrenal gland without perilesional stranding or intralesional haemorrhage, which measured $3.2 \times 2.6 \times$ $2.5 \mathrm{~cm}$. MRI brain showed normal pituitary gland. On the basis of all above investigations, adrenal adenoma was suspected and patient underwent laparotomy for removal of mass. Histopathological examination of adrenal mass showed trabecular architecture with broad fibrous bands. The neoplastic cells had pink granular cytoplasm with some cells showing empty cytoplasmic appearance. There was evidence of brown pigmentation and fibrovascular septae (Fig. 1). These all features were suggestive of adrenocortical neoplasm. Tissue was further examined by immunohistochemistry to differentiate from adrenal cortical hyperplasia. Immunohistochemical examination revealed immunoreactive for cytokeratins (AE1/AE3 and CAM5.2). Inhibin was focally positive. On the basis of 
Figure 1

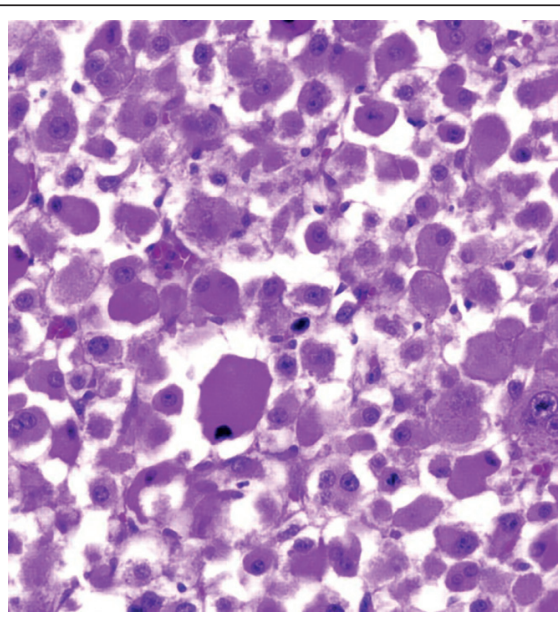

Neoplastic cells with pink granular cytoplasm and brown pigmentation.

these findings, the final diagnosis of an adrenocortical oncocytoma (according to the WHO classification of adrenal cortical adenoma oncocytoma) was made. The patient is under clinical observation with repeated ultrasound and CT examination, finding no recurrence or metastases within 2 years after the surgery.

\section{Discussion}

The widespread use of abdominal ultrasonography, computed tomography and MRI has resulted in a frequent clinical problem of having to evaluate the potential malignancy or hormonal activity of an adrenal mass. The evaluation of an adrenal mass includes hormonal activity and radiologic characteristics of the tumour, as well as a history of previous malignant lesions [2]. Oncocytomas are tumours in which all or almost all of the cells are oncocytic. Ultrastructural study reveals oncocytes containing numerous mitochondria. Neoplasms, composed predominantly of oncocytes, are well defined in the kidney, thyroid and salivary glands. There have been reports of very rare oncocytic tumours in the pituitary gland, parathyroid glands, lacrimal glands and spinal cord [3]. An adrenocortical localization of oncocytoma is diagnosed also very rarely. These tumours are mostly nonfunctioning and benign. The tumours are detected mainly incidentally during investigation of unrelated symptoms [5]. In our case, the tumour was found during workup of Cushing syndrome. The size of the mass and imaging phenotype are the two major predictors of malignant disease. The latest guidelines for the management of adrenal tumours, published in 2009, recommend adrenalectomy after hormonal evaluation in patients with a tumour of at least $4 \mathrm{~cm}$ in diameter, although the patient's age as well as coexisting conditions should be taken into account.

The pathological examination of this tumour was based on the Weiss scale, in which nuclear grade atypia, the mitotic rate, atypical mitoses, the character of cytoplasm, the architecture of the tumour, necrosis, veins, sinusoids and tumour capsule infiltration were considered. The presence of at least four of these nine criteria is indicative of malignancy. Bisceglia et al. proposed Weiss modified criteria indicating malignant or benign lesions and tumours of uncertain malignant potential [6]. If the tumour presents any of the major criteria (high mitotic activity, atypical mitoses or venous invasion), it is considered malignant; if the tumour presents any of the minor criteria (large size, necrosis, capsular or sinusoidal invasion), it is considered to have uncertain malignant potential and none of these features indicates a benign tumour. Our patient was diagnosed as having an oncocytic neoplasm, with malignant potential. The patient underwent clinical observation with repeated ultrasound and computed tomographic examination, finding no recurrence or metastases within 2 years after the surgery.

\section{Conclusion}

Adrenocortical oncocytoma, although extremely rare, should be considered as a possible diagnosis in adrenal tumours. Although most of these tumours are nonfunctioning, they can be hypersecreting also.

\section{Acknowledgements \\ Conflicts of interest}

There are no conflicts of interest.

\section{References}

1 Hamperi $\mathrm{H}$. Contributions to normal pathological and histological human saliva drusen. Z Microanaf researchers 1931;27:1.

2 Chang A, Harawi SJ. Oncocytes, oncocytosis, and oncocytic tumors. Pathol Annu 1992; 27:263-304.

3 Rouzbahman M, Serra S, Chetty R. Rectal adenocarcinoma with oncocytic features: possible relationship with preoperative chemoradiotherapy. J Clin Pathol 2006; 59:1039-1043.

4 Vancura RW, Thomas JH, Jewell WR, Damjanov I. Bilateral oncocytic malignant melanoma in axillary lymph nodes without evidence of an extranodal primary. Ultrastruct Pathol 2005; 29:399-404.

5 Radi MJ, Fenoglio-Preiser CM, Chiffelle T. Functioning oncocytic islet-cell carcinoma. Report of a case with electron-microscopic and immunohistochemical confirmation. Am J Surg Pathol 1985; 9:517-524

6 Bisceglia M, Ludovico O, Di Mattia A, Ben-Dor D, Sandbank J, Pasquinelli G, et al. Adrenocortical oncocytic tumors: report of 10 cases and review of the literature. Int J Surg Pathol 2004;12:231-243. 